Western University

Scholarship@Western

9-2-2016

\title{
The prevalence, location, severity, and daily impact of pain reported by youth and young adults with cerebral palsy.
}

Laura Brunton

Sarah Hall

Adrienne Passingham

Julie Wulff

Rich Delitala

Follow this and additional works at: https://ir.lib.uwo.ca/ptpub

Part of the Physical Therapy Commons

Citation of this paper:

Brunton, L., Hall, S., Passingham, A., Wulff, J., \& Delitala, R. (2016). The prevalence, location, severity, and daily impact of pain reported by youth and young adults with cerebral palsy. Journal of pediatric rehabilitation medicine, 9(3), 177-183. 
The Prevalence, Location, Severity, and Daily Impact of Pain Reported by Youth and Young Adults with Cerebral Palsy.

Laura Brunton ${ }^{1} \mathrm{PhD}$, MPT, Sarah Hall ${ }^{2}$ MPT, Adrienne Passingham ${ }^{2}$ MPT, Julie Wulff ${ }^{2}$ MPT, Rich Delitala $^{2}$ MPT

${ }^{1}$ Faculty of Kinesiology, University of Calgary, Calgary, Alberta, Canada

${ }^{2}$ School of Physical Therapy, Western University, London, Ontario, Canada

Corresponding Author: Laura Brunton PhD, MPT

Email: 1brunto3@uwo.ca 
Abstract

PURPOSE To describe the prevalence, location, severity, and daily impact of pain reported by youth and young adults with cerebral palsy $(\mathrm{CP})$. A secondary aim was to identify any significant associations between the constructs of interest.

METHOD An observational study of 112 participants with CP to understand their experience of pain through a questionnaire. Participants were 56 males and 55 females with a mean age of $18 \mathrm{y} 9 \mathrm{mo}$ (SD 4y 5mo).

RESULTS Pain was reported by $75 \%$ of males and $89 \%$ of females. Both severity and impact of pain were significantly greater in females. In addition, severity and impact of pain were significantly different between specific GMFCS levels. There were no significant differences in location of pain by gender or GMFCS level. A strong positive correlation between the severity and impact of pain was observed $\left(r_{s}=0.80\right)$.

CONCLUSION The gender differences in the severity and impact of pain and the overall and high prevalence of pain reported here provide healthcare practitioners with an increased awareness of pain distribution/characteristics among young adults with CP. Understanding the impact of pain on daily life can assist practitioners to efficiently manage pain and improve the quality of life for individuals with $\mathrm{CP}$.

Key words: Cerebral Palsy; Pain; Prevalence; Severity; Impact; Youth; Young Adult. 


\section{Introduction}

The transition from adolescence to adulthood presents new demands and as a result requires increased independence. (1) For individuals with cerebral palsy $(\mathrm{CP})$, health care becomes more fragmented in adulthood and these individuals are required to take a more active role in managing their own care. For this population, this time of transition can be marked by a significant decrease in function, including an increased prevalence of secondary conditions such as pain and fatigue. (2) It is therefore essential that these secondary conditions are appropriately described, identified, managed and prevented in youth and young adults with CP. (2-3)

The experience of pain affects people with CP to varying degrees. (1, 3-5) Research suggests that between 24 to $83 \%$ of individuals with CP report having pain (depending on the study sample), (3, 6-8) with the legs and feet, low back, hip, and the upper extremities being the most frequently reported location. (3, 8-9) Malone and Vogtle (3) identified a higher number of painful sites in individuals who were ambulatory; however, factors such as age and gender were not shown to affect the location of pain. (3) Age, specifically over 14 years, has been shown to be a significant predictor for the presence of pain in individuals with CP. (5, 9-10) Additionally, an increased prevalence of secondary conditions has been observed with increasing age, (2) and as a result, adults report significantly higher levels of pain and greater incidences of recurrent chronic pain when compared to children with CP. (8-11) In a study describing the intensity of pain in individuals with $\mathrm{CP}$ between the ages of 18 to $74,23 \%$ of participants identified pain levels of 7 or greater on a visual analog scale (range 0-10). (5) Previous studies have shown that women and individuals with quadriplegic involvement have higher reported incidences of pain; however, these findings have been inconsistent. $(2-3,11)$ Although the pain experience may 
change as a result of varying functional abilities, the overall incidence of chronic pain has not been found to differ significantly among individuals across all Gross Motor Function Classification Scale (GMFCS) levels. (3, 5-6)

Pain has been reported as a primary concern for many individuals with $\mathrm{CP}(7)$ and has been shown to affect participation in self-care, leisure, productivity, rest and health-related quality of life. $(3,10)$ In addition, the presence of pain within this population can result in an impact on independence and performance of daily activities that ranges from moderate to extreme, depending on the individual pain experience. (4) Furthermore, pain is often related to increased emotional disturbance which includes frustration, loneliness, distress and desperation. $(1,12-14)$ There is limited evidence describing the magnitude of the relationship between pain intensity and the impact of pain on activities of daily living within this population.

Although pain is of significant concern for many individuals living with $\mathrm{CP},(7)$ current evidence focuses on children and youth or adults with CP. There is a lack of quantitative analyses and detailed descriptions of the pain experience during the transition from late adolescence to young adulthood. (12) With such knowledge of this pain experience health care practitioners will be better able to support the needs of these individuals through their transition to adulthood and beyond. In addition, with use of prophylactic, long-term, self-management strategies, adults with CP may be able to reduce the incidence, severity and impact of secondary conditions including pain and thereby increase their quality of life. $(2,12-13,15)$ The objectives of this study were to describe the prevalence, location and severity of pain, and the impact it may have on the daily lives of youth and young adults with cerebral palsy aged 14-31 years. A secondary objective of this study was to identify potential significant association between the constructs investigated. 


\section{Method}

\subsection{Design}

This was a cross-sectional, observational study with descriptive purposes. A convenience sample was used to assess pain in individuals with CP through a questionnaire, which was either completed online or returned by mail between March and December 2012. Ethical approval for this study was obtained by the Health Science Review Ethics Board at Western University.

\subsection{Participants}

The inclusion criteria consisted of individuals diagnosed with CP aged 14 to 31 who were able to communicate through verbal or written response to the questionnaire. Nineteen children treatment centres across Ontario were contacted for participation in the study. Of these, six centres agreed to participate in the study by identifying eligible clients from their databases and facilitated the mailing of questionnaire packages. The mailing procedure followed a modified Dillman method. (16) In total, seventy-five participants were recruited through the children centres. Returning a completed questionnaire was considered implied consent to participate in the study.

Twenty-five participants were recruited through advertisements posted in existing Facebook groups for individuals living with CP. Eight individuals with $\mathrm{CP}$ were also recruited from a previous study from which participants provided consent to be contacted for further research. An additional four participants responded to a study advertisement posted on the Ontario Foundation of Cerebral Palsy (OFCP) website and newsletter.

Through the various methods of recruitment, 314 potential participants were contacted and 135 responded to the study request (43\% response rate). Twenty-three returned questionnaires were not included in the analysis. Eight individuals returned the questionnaires 
blank and did not want to participate in the study, another 8 questionnaires were completed entirely by parental proxy and 7 questionnaires were returned blank with instructions to the study team that the participants were unable to communicate or comprehend the questionnaire and thus were not eligible for the study. As a result, a total of 112 participants who responded to the study request were included in data analysis. Table 1 contains the demographic characteristics of the sample.

\subsection{Measures}

The data analyzed in this study is a subset of a larger study focused on fatigue. The relevant measures for the purpose of this study are the Gross Motor Function Classification System Expanded and Revised Version (GMFCS-ER) and a pain questionnaire originally reported by Doralp and Bartlett. (14) The GMFCS-ER has established validity and was used in this study to classify individuals over the age of 12 with respect to their level of motor function. (17-18) This tool was selected for use beyond the validated age in this study for consistency of sample description.

The pain questionnaire is a short measure used to identify the prevalence, location, severity, and impact of pain on daily life for individuals with CP. The first question asks, "Over the past month, have you experienced physical pain". If the individual selected "NO", then the questionnaire was complete. If the individual selected "YES", they were to answer further questions addressing their pain location, severity and the impact of pain on daily life. The individual selected specific areas of the body that were painful over the past month from a diagram of the body, regions included the: neck, shoulder, elbow/forearm, upper back, lower back, wrist/hand, hip, thigh, knee, calf, ankle/foot. The average severity of their pain was rated from 1 (very little pain) to 10 (extremely painful). The daily impact of the pain was rated on a 
scale from 1 (does not get in the way at all) to 10 (unable to carry out activities). The pain questionnaire has been show to have moderate levels of test-retest reliability for all dimensions.

\subsection{Statistical Analysis}

Data were analyzed using SPSS for Windows (IBM Corp. Released 2012. IBM SPSS Statistics for Windows, Version 21.0. Armonk, NY: IBM Corp). Ordinal level data were represented by mode, median and range. The differences in pain prevalence, separated by both gender and GMFCS level, were analyzed using Chi-square tests. The differences in distribution of painful body regions, by both gender and GMFCS level, were calculated using a Chi-Square test with a Bonferroni correction for multiple comparisons. The severity and impact of pain were both analyzed for differences between genders using the Mann-Whitney U and between GMFCS levels using the Kruskall-Wallis 1-way ANOVA. Associations among the four constructs of prevalence, body region, severity and impact were identified using Spearman's rho. For all analyses, a $p$ value of $<0.05$ was considered statistically significant. In the case of missing data (Gender or GMFCS level) the participants' data was excluded from the subgroup analyses.

3. Results

In this study, pain was reported by $75 \%$ of male and $89 \%$ of female respondents (Figure 1). There was no statistically significant difference in the prevalence of pain between genders $(p=0.053)$ or among GMFCS levels ( $p=0.68)$ (Figure 1). Of those who did experience pain,

youth and young adults in GMFCS level V experienced pain most often (88\%) and individuals in GMFCS Level III had the lowest reported prevalence of pain (70\%).

There was no significant difference among number of painful body regions between genders $(p>0.05)$, however, a greater number of females reported experiencing pain at each body 
site when compared to males (Figure 2). There were no significant differences among painful body regions amongst GMFCS levels $(p>0.05)$ (Figure 2). Greater than half of the participants who reported pain identified the lower back (55\%) and ankle/foot (52\%) as painful regions. Youth and young adults in GMFCS level I reported neck (26\%) and calf (61.5\%) pain more often than participants of other GMFCS levels. Those in GMFCS level II reported experiencing pain in the greatest number of body regions, which included shoulder (38\%), elbow/forearm (17\%), wrist/hand (30\%), lower back (63\%), hip (50\%), thigh (27\%), knee (57\%), and ankle/foot (63\%). Of all participants reporting pain in the upper back, those of GMFCS level III and IV reported the highest incidence $(n=7,50 \%$ and $n=7,54 \%$ respectively).

The severity of pain among females was significantly higher than males $(p=0.003)$, with a median of 6/10 for females and 3/10 for males (range 1-10) (Figure 3). A significant difference was observed for the severity of pain among GMFCS levels (Figure 3), post-hoc analysis revealed the difference to exist only between GMFCS level I and III. The median severity of pain was lowest in GMFCS Level I (median 3, range 1-8) and highest in GMFCS level III (median 6, range 2-10).

Of those who experienced pain, $98 \%$ of females and $95 \%$ of males reported that, within the past month, their pain impacted their daily activities (Figure 3). The impact of pain was significantly greater in females compared to males $(p=0.014)$, with median values of $5 / 10$ for females and 3/10 for males (range 1-10). There was a significant difference for the impact of pain among GMFCS levels ( $p=0.04$ ) (Figure 3). Post-hoc analysis revealed that the difference existed only between GMFCS level I and II, such that individuals classified as GMFCS level II experienced a greater impact of pain. In addition, there was a strong positive correlation between the severity of pain and its impact on activities of daily living $\left(\mathrm{r}_{\mathrm{s}}=0.80\right)$. 


\section{Discussion}

In this study, over $75 \%$ of respondents reported experiencing pain within the last month. This is consistent with another non-population based study by Hirsh and colleagues, (5) which identified the prevalence of pain as 73\% in adult respondents. (5) Such findings demonstrate a higher prevalence than those previously reported by Dickinson and colleagues, (20) and Doralp and Bartlett, (15) both of whom reported on population-based samples. As a result, respondents in the current study may have been more likely to experience pain when compared to the whole population of individuals with CP; and may be a limitation of this study. However, it should also be noted that there may be other factors influencing pain in these young adults. In other studies, individuals with $\mathrm{CP}$ over 14 years of age have been shown to have a significantly greater prevalence of pain, $(5,9-10)$ supporting the current findings and potentially hinting to an effect of age and the maturation process on the pain experience. Many transition programs begin around age 14 and the increasing independence in these young adults could be leading to other physical, psychological, or social demands that may affect the pain experience. Future studies need to comprehensively collect outcomes across all levels of the life experience of cerebral palsy to understand how pain is truly impacting these individuals.

In this study, females reported experiencing more pain than males, at $89 \%$ and $75 \%$ respectively; however, it did not reach statistical significance. Previous research has shown females to have a higher incidence of pain, $(2-3,11)$ consistent with the trend demonstrated in the current study. Additionally, the results of this study agree with previous research which has shown that the prevalence of pain does not significantly differ among GMFCS levels. $(3,5-6)$ This study did not identify any significant difference among the distribution of painful body regions either between genders or among GMFCS levels. The study by Doralp and Bartlett (15) 
identified similar non-significance among painful body regions for both gender and GMFCS in children and youth with CP. (15) Results from the current study related to the overall prevalence of pain per body region was consistent with previous research, identifying that pain was most prevalent in the low back and ankle/foot regions. $(4,7,21)$ Visual inspection of the responses in this study indicated that individuals in GMFCS level I report pain most frequently in the neck and calf; individuals in GMFCS level II report more areas of pain when compared to all other levels; individuals in GMFCS levels III and IV report pain most frequently in the upper back region. Although the results were non-significant, these pain characteristics are important to consider, as they will help to identify and describe the relationship between functional abilities and the pain experience for individuals with CP. Such understanding will help to direct effective treatment and pain self-management within this population. Overall, research concerning painful body regions in individuals with $\mathrm{CP}$ has not been consistent or conclusive and needs to be studied further with larger numbers to allow for subgroup analyses within the distinct GMFCS levels.

The severity of pain was significantly different between males and females within this study, with females experiencing more severe pain than males with a median of $6 / 10$ and 3/10, respectively. To date, there has been no research demonstrating a difference in severity of pain experienced between genders for individuals with $\mathrm{CP}$; however, the pain experience has been shown to differ between males and females within non-CP populations. (22) In other populations, females have been shown to have greater sensitivity to pain and less pain inhibition than males. When GMFCS levels were analyzed separately the only significant difference was observed between GMFCS level I and III. Individuals classified as GMFCS level III tend to have relative functional independence (23); however, having significant physical impairments that 
may predispose them to further secondary conditions, including pain. $(2,12-13,15)$ The results of this study differ from previous findings from Doralp and Bartlett, (15) which identified children and youth of GMFCS levels IV and V as having the most severe pain. It is not clear whether these observed differences are as a result of differing sample characteristics or as a result of participant age differences between the two studies. This should be addressed in future research.

The overall impact on daily life was reported as significantly greater in females when compared to males. Although pain has been shown in previous research to have an impact on daily life of individuals with $\mathrm{CP},(1,3-4)$ no research to date has identified a difference in the impact of pain between genders. (3) This study also identified a significant difference between GMFCS levels I and II, related to the impact of pain on daily life. This indicates that individuals with different levels of functional impairment are impacted to varying degrees as a result of their pain experience. Future research should further investigate how the pain experience specifically impacts individuals at each GMFCS level.

The current findings show that females report significantly higher severity and impact of pain, when compared to males. The severity and impact of pain were shown to have a strong, positive correlation, indicating that individuals with more severe pain reported a greater impact of that pain on their daily lives. Although both constructs have been studied separately within the $\mathrm{CP}$ population, this is the first study to demonstrate the association between the severity and impact of pain in youth and young adults. Such information is essential to outline the importance of pain management for individuals with $\mathrm{CP}$.

\subsection{Limitations}


The primary limitation of this study is that the sample was generated through convenience sampling and may not be representative of the population of individuals with $\mathrm{CP}$. This could limit the generalizability of the results; however, the prevalence estimates are similar to those identified in population based studies. Future research should identify whether the current findings of the pain experience in $\mathrm{CP}$ can be reproduced within a population-based sample. It is possible that other significant differences exist between GMFCS levels but did not reach statistical significance in this study due to the small sample size in each GMFCS level. In addition, the majority of our study respondents were characterized as GMFCS level I, II or III with fewer individuals in levels IV and V, thereby further decreasing the power of the study to detect differences related to functional ability. Future research with larger sample sizes should be conducted to fully elucidate differences in pain severity and impact related to functional ability.

\section{Conclusion}

The findings of this study are important to describe the pain experience and its effect on daily life of youth and young adults with CP. This study has identified gender differences in the pain experience and has outlined a strong relationship between pain severity and its impact on daily life for individuals with CP. Such information will enable healthcare professionals to explore and gain a further understanding of the characteristics of pain in $\mathrm{CP}$, with emphasis on efficiently managing pain, to ultimately to improve the quality of life for individuals with CP.

Acknowledgements: The authors would like to thank Doreen Bartlett, Western University, for her review of this paper as well as her guidance and support of this project. This study was supported by a Canadian Doctoral Student Scholarship awarded to L. Brunton from the Canadian Institutes of Health Research. The funding agency had no role in study design, data collection, analysis or preparation of the manuscript and no influence on publication decisions.

Statement of Conflict of Interest: The authors of this paper report no conflicts of interest. 


\section{REFERENCES}

1. Castle K, Imms C, Howe L. Being in pain: A phenomenological study of young people with cerebral palsy. Dev Med Child Neurol. 2007; 49(6): 445-449.

2. Gajdosik CG, Cicirello N. Secondary conditions of the musculoskeletal system in adolescents and adults with cerebral palsy. Phys Occup Ther Pediatr. 2001; 21(4): 49-68.

3. Malone LA, Vogtle LK. Pain and fatigue consistency in adults with cerebral palsy. Disabil Rehabil. 2010; 32(5): 385-391.

4. Jahnsen R, Villien L, Aamodt G, Stanghelle JK, Holm I. Musculoskeletal pain in adults with cerebral palsy compared with the general population. J Rehabil Med. 2004; 36(2): $78-84$.

5. Hirsh AT, Gallegos JC, Gertz KJ, Engel JM, Jensen MP. Symptom burden in individuals with cerebral palsy. J Rehabil Res Dev. 2010; 47(9): 863-876.

6. Van Der Slot WM, Nieuwenhuijsen C, Van Den Berg-Emons RJ, et al. Chronic pain, fatigue, and depressive symptoms in adults with spastic bilateral cerebral palsy. Dev Med Child Neurol. 2012; 54(9):

7. Engel JM, Jensen MP, Hoffman AJ, Kartin D. Pain in persons with cerebral palsy: extension and cross validation. Arch Phys Med Rehabil. 2003; 84(8):1125-1128.

8. Engel JM, Petrina TJ, Dudgeon BJ, McKearnan KA. Cerebral palsy and chronic pain: a descriptive study of children and adolescents. Phys Occup Ther Pediatr. 2005; 25(4): 73 84.

9. Ramstad K, Jahnsen R, Skjeidal OH, Diseth TH. Characteristics of recurrent musculoskeletal pain in children with cerebral palsy aged 8 to 18 years. Dev Med Child Neurol. 2011; 53(11): 1013-8. 
10. Findlay B, Switzer L, Narayanan U, Chen S, Fehlings D. Investigating the impact of pain, age, Gross Motor Functional Classification System, and sex on health-related quality of life in children with cerebral palsy. Dev Med Child Neurol. 2015; epub DOI: 10.1111/dmcn.12936.

11. Riquelme I, Cifre I, Montoya P. Age-related changes of pain experience in cerebral palsy and healthy individuals. Pain Med. 2011; 12(4): 535-45.

12. Engel JM, Jensen MP, Schwartz L. Coping with chronic pain associated with cerebral palsy. Occup Ther Int. 2006; 13(4): 224-33.

13. Jensen MP, Engel JM, Schwartz L. Coping with cerebral palsy pain: a preliminary longitudinal study. Pain Med. 2006; 7(1): 30-7.

14. Liptak GS. Health and well being of adults with cerebral palsy. Curr Opin Neurol. 2008; 21(2): $136-42$.

15. Doralp S, Barlett DJ. The prevalence, distribution, and effect of pain among adolescents with cerebral palsy. Pediatr Phys Ther. 2010; 22(1): 26-33.

16. Dillman DA. Mail and internet surveys: The tailored design method. 2nd ed. New York, NY: John Wiley \& Sons Inc; 2000.

17. Palisano RJ, Rosenbaum P, Bartlett D, Livingston MH. Content validity of the expanded and revised Gross Motor Function Classification System. Dev Med Child Neurol. 2008; 50(10): 744-50.

18. Gorter JW, Slaman J, Bartlett D, Van den Berg-Emons HJG. Reliability of the gross motor function classification system expanded and revised (GMFCS-ER) when used with adolescents and young adults with cerebral palsy. Dev Med Child Neurol. 2011; 53(s5): $42-43$. 
19. Brunton LK. Measuring fatigue in adolescents and young adults with cerebral palsy. University of Western Ontario - Electronic Thesis and Dissertation Repository. Paper 2133; 2014. http://ir.lib.uwo.ca/etd/2133

20. Dickinson HO, Parkinson KN, Ravens-Sieberer U, et al. Self-reported quality of life of 812-year-old children with cerebral palsy: a cross-sectional European study. Lancet. 2007; 369(9580): 2171-8.

21. Schwartz L, Engel JM, Jensen MP. Pain in persons with cerebral palsy. Arch Phys Med Rehabil. 1999; 80(10):1243-6.

22. Bartley EJ, Fillingim RB. Sex differences in pain: a brief review of clinical and experimental findings. J. Anaesth. (2013) 111 (1): 52-58 .

23. Tarsuslu T, Livanelioglu A. Relationship between quality of life and functional status of young adults with cerebral palsy. Disabil Rehabil. 2010; 32: 1658-1665. 
Table 1 - Participant Demographics

\begin{tabular}{|c|c|c|c|}
\hline Characteristic & Female & Male & Total \\
\hline Sex* n, $(\%)$ & $55(50)$ & $56(50)$ & 111 \\
\hline Age mean (SD) & $20.00(5.04)$ & $17.50(3.38)$ & $18.73(4.43)$ \\
\hline \multicolumn{4}{|l|}{$\begin{array}{l}\text { Distribution** } \mathrm{n} \\
(\%)\end{array}$} \\
\hline Monoplegia & $2(4)$ & $4(8)$ & $6(6)$ \\
\hline Hemiplegia & $13(24)$ & $16(31)$ & $29(27)$ \\
\hline Diplegia & $19(34)$ & $18(34)$ & $37(35)$ \\
\hline Triplegia & $6(11)$ & $3(6)$ & $9(8)$ \\
\hline Quadriplegia & $15(27)$ & $11(21)$ & $26(24)$ \\
\hline Total & $55(100)$ & $52(100)$ & $107(100)$ \\
\hline \multicolumn{4}{|l|}{$\begin{array}{l}\text { GMFCS Level }^{\circ} \mathrm{n} \\
(\%)\end{array}$} \\
\hline I & $13(24)$ & $20(36)$ & $33(30)$ \\
\hline II & $16(29)$ & $18(32)$ & $34(31)$ \\
\hline III & $12(22)$ & $8(14)$ & $20(18)$ \\
\hline IV & $9(16)$ & $7(13)$ & $16(14)$ \\
\hline V & $5(9)$ & $3(5)$ & $8(7)$ \\
\hline Total & $55(100)$ & $56(100)$ & $111(100)$ \\
\hline \multicolumn{4}{|c|}{$\begin{array}{l}\text { *Sex was not reported by one } \\
\text { participant } \\
\text { ** Distribution was not reported by } \\
\text { five participants } \\
{ }^{\circ} \text { GMFCS Level was not reported } \\
\text { by one participant }\end{array}$} \\
\hline
\end{tabular}


Figure Legends

Figure 1. The prevalence of pain by gender and GMFCS level

Figure 2. Proportion of individuals who reported pain in specific body regions by gender and GMFCS level. N indicates neck; Sh, shoulder; E/F, elbow and forearm; W/H, wrist and hand; $\mathrm{H}$, hip; Th, thigh; Kn, knee; Ca, calf; A/F, ankle and foot; UB, upper back; LB, lower back.

Figure 3. The severity and daily impact of pain by gender and GMFCS level 
Figure 1

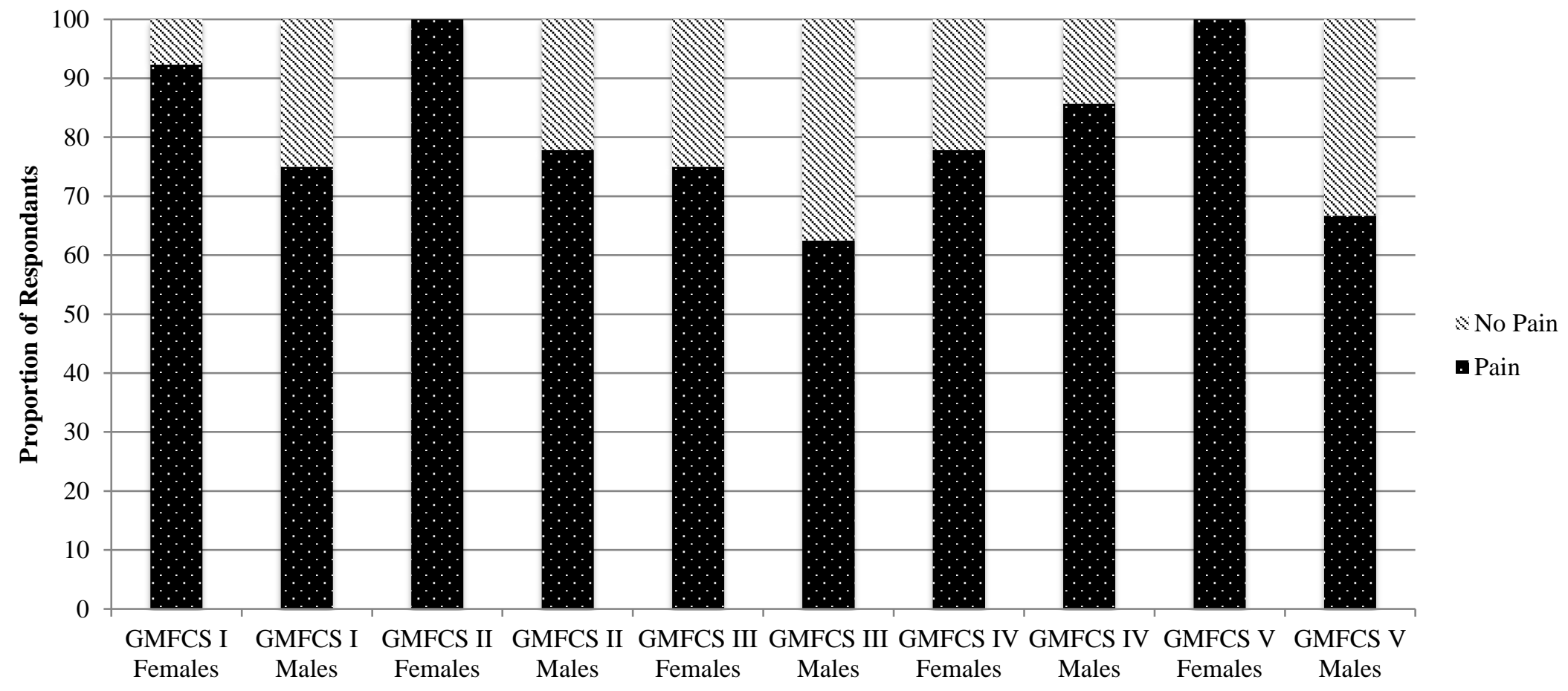


Figure 2

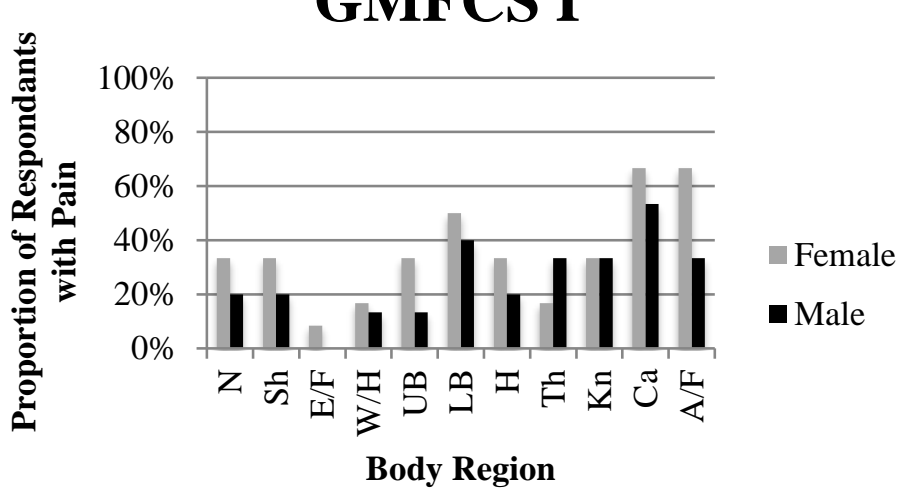

GMFCS III

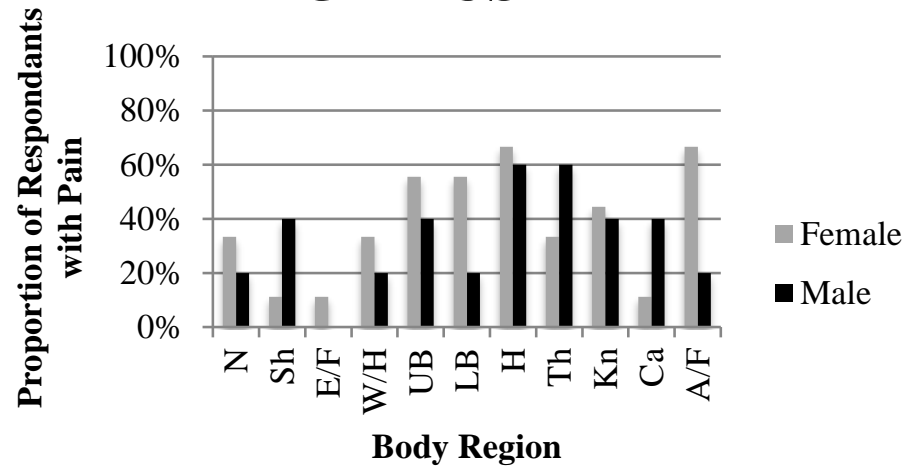

GMFCS II

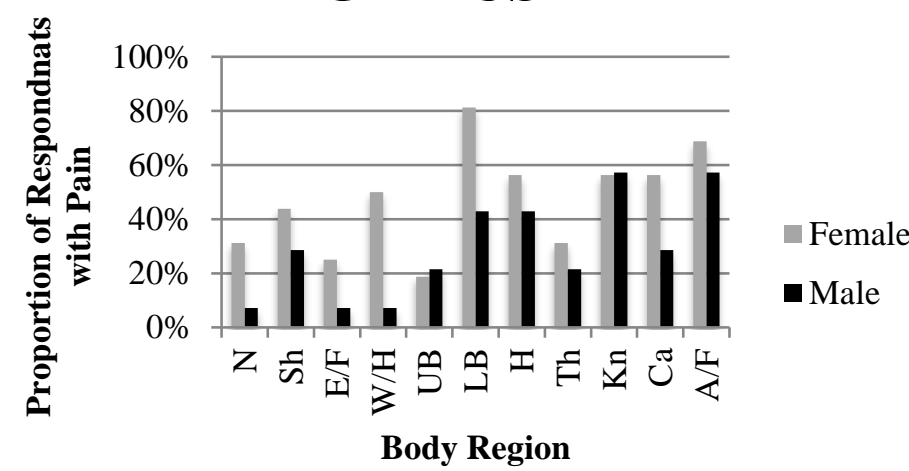

GMFCS IV

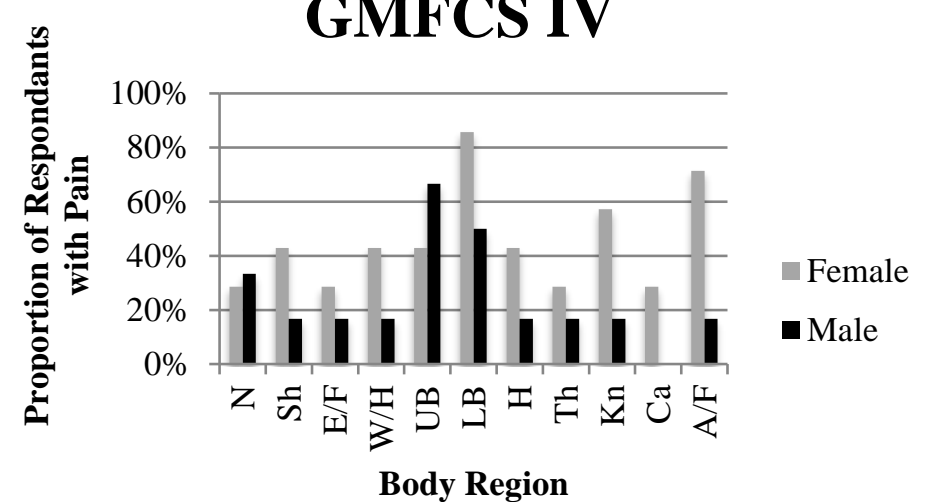

GMFCS V

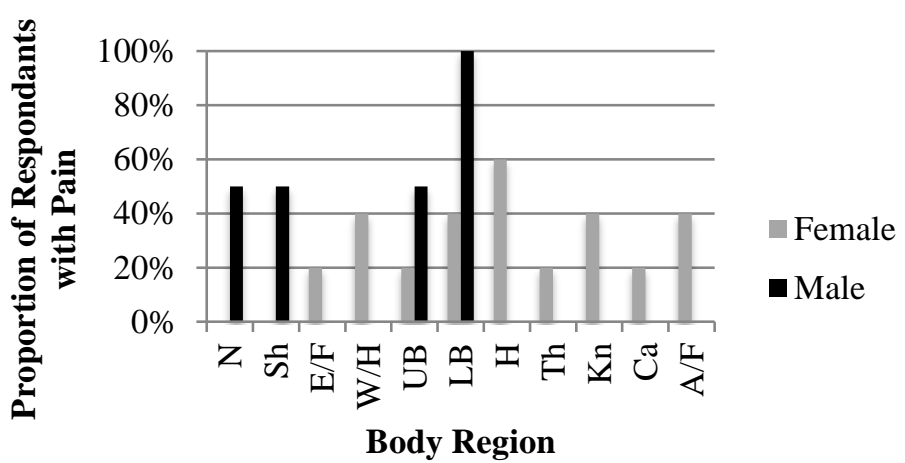


Figure 3
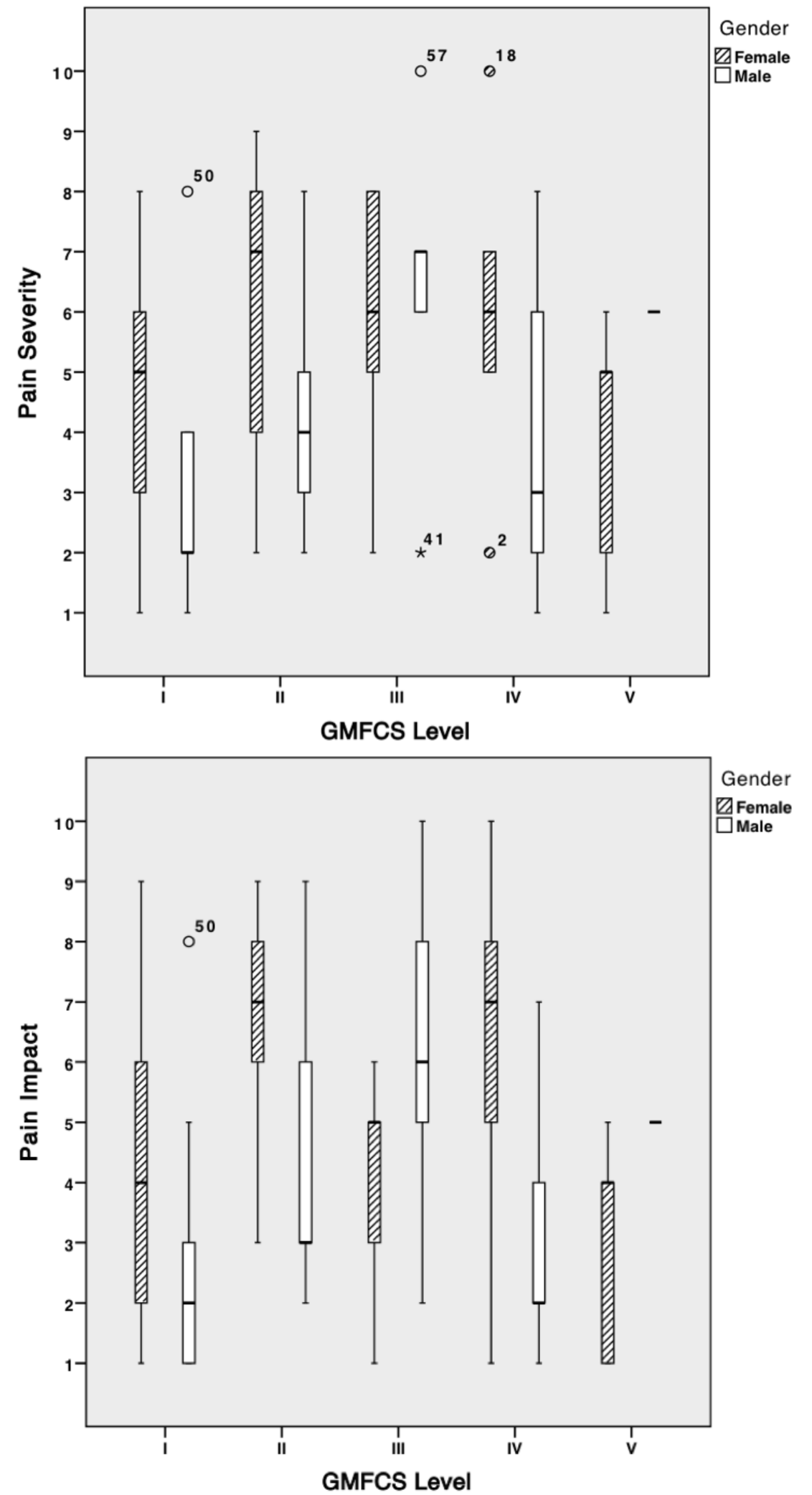